\section{Factors associated with recurrent Plasmodium vivax malaria in Porto Velho, Rondônia State, Brazil, 2009}

\author{
Fatores associados às recidivas de malária causada \\ por Plasmodium vivax no Município de Porto \\ Velho, Rondônia, Brasil, 2009
}

Luciano Rodrigues Simões 1,2 Eduardo Rodrigues Alves-Jr 1 Daniele Ribatski-Silva 1 Luciano Teixeira Gomes 1 Andréia Ferreira Nery 1 Cor Jesus Fernandes Fontes 1,3

\author{
1 Faculdade de Medicina, \\ Universidade Federal de \\ Mato Grosso, Cuiabá, Brasil. \\ 2 Faculdade de Cuiabá, \\ Cuiabá, Brasil. \\ ${ }^{3}$ Faculdade de Medicina, \\ Faculdade de Ciências \\ Médicas de Cacoal, Cacoal, \\ Brasil. \\ Correspondence \\ C. J. F. Fontes \\ Núcleo de Estudos de \\ Doenças Infecciosas e \\ Tropicais de Mato Grosso, \\ Faculdade de Medicina, \\ Universidade Federal de \\ Mato Grosso. \\ Rua Tulipas, 316, Cuiabá, MT \\ 78049-412, Brasil. \\ corfontes@gmail.com
}

\begin{abstract}
This open retrospective cohort study aimed to describe the incidence of recurrent Plasmodium vivax malaria and associated factors in Porto Velho, Rondônia State, Brazil, in 2009. Data were collected from the National Information System for Malaria Epidemiological Surveillance. There were 23,365 reported $\mathrm{P}$. vivax malaria cases in 2009, 23\% of which were classified as relapses. Incidence density of $\mathrm{P}$. vivax recurrence was 45.1/100 patient-years, mostly occurring between the 4th and 13th week after initiating treatment. Male gender, shorter time since onset of symptoms, and higher parasitemia in the initial infection increased the risk of relapse during the year, with a $10 \%$ reduction in relative risk for longer symptoms and $11 \%$ and $15 \%$ increases in relative risk for males and higher initial parasitemia, respectively. However, the results show low clinical relevance for these associations, thereby limiting their applicability to decision-making at the public health level.
\end{abstract}

Recurrence; Plasmodium vivax; Malaria

\section{Resumo}

Estudo de coorte histórica aberta, construída com informações registradas no Sistema de Informação de Vigilância Epidemiológica da Malária, com objetivo de descrever a incidência e os fatores associados à recidiva de malária causada pelo Plasmodium vivax em Porto Velho, Rondônia, Brasil. Foram notificados 23.365 casos de malária por $\mathrm{P}$. vivax no município, sendo que $23 \%$ deles apresentaram recidiva no decorrer de 2009. A densidade de incidência de recidivas foi de 45,1/100 pacientes-ano, ocorrendo, principalmente, entre a $4 \underline{a}$ e a $13 \underline{a}$ semana após o início do tratamento. Ser homem, ter menos tempo de sintomas e ter maior nível de parasitemia na infecção inicial aumentaram o risco de recidiva no decorrer do ano, com $10 \%$ de redução relativa do risco para maior tempo de sintomas e de $11 \%$ e $15 \%$ de incremento relativo do risco para o sexo masculino e maiores níveis de parasitemia, respectivamente. Contudo, os resultados demonstram baixa relevância clínica das associações encontradas, comprometendo a sua aplicabilidade na tomada de decisão em nível de saúde pública.

Recidiva; Plasmodium vivax; Malária 


\section{Introduction}

The Brazilian Ministry of Health recorded 293,794 cases of malaria in the country in 2011, 99.9\% of which in the Legal Amazonia. Of this total, 257,172 (87.5\%) were caused by Plasmodium vivax, and the highest incidence rates were in the States of Pará (44.5\%), Amazonas (21.8\%), and Rondônia (12.8\%). Rondônia had 42,761 malaria cases in the year 2010, representing $13.1 \%$ of all cases reported in Brazil that year. $P$. vivax was the most prevalent species in the region, with $89 \%$ of cases, followed by Plasmodium falciparum with $10.1 \%$ and less than $1 \%$ of mixed infections. Annual malaria incidence in the Municipality (County) of Porto Velho has reached high levels, ranging from 20 to 400 cases per 1,000 inhabitants 1 .

Recurrent malaria is defined as recurrent asexual parasitemia following treatment of the disease, after having observed negative parasitemia during a varied period of time 2 . Recurrence is due to one of the following: (a) therapeutic failure resulting from non-adherence to treatment 3 , resistance of the parasite to the drugs used 4, poor quality of the medication 5 , or sub-therapeutic doses of the drugs 4; (b) reactivation of hypnozoites 2; and (c) exposure to new infection by the mosquito vector. Conceptually, recurrent malaria can result from recrudescence, relapse, or reinfection.

Recrudescence of malaria occurs when the bloodstream forms of the parasite are not completely eradicated by treatment and re-expand in number after a decline in serum concentration of the drugs. This occurs more frequently in malaria from P. falciparum and P. vivax and rarely with P. malariae 6 .

Relapse is defined as resurgence of parasitemia and clinical manifestations due to reinvasion of the erythrocytes by merozoites from dormant hypnozoites in the liver. Relapses are believed to occur 21 to 140 days after treatment of the tropical strain and 180 to 420 days after treatment of the temperate strain ${ }^{2}$. The main cause is treatment failure. Based on clinical observation alone, it is very difficult to distinguish recrudescence from relapse or relapse from reinfection. In some situations the distinction can be made by identifying a parasite genotype in the relapse which is identical to that of the primary infection. Still, genotyping parasites from the initial infection and relapse has not proven efficient in distinguishing between relapse and reinfection 6,7,8,9,10,11 .

Reinfection with P. vivax is a new infection acquired by patients treated for malaria after a time interval inconsistent with recrudescence or relapse. In genotyping, reinfection can be defined by finding a parasite that is genetically different from the one that caused the primary infection 12 . Since the intervals between episodes overlap, even with genotyping it is also difficult to distinguish precisely between relapse and reinfection.

Studies have agreed that up to 28 days, when there is still drug circulating after treatment, recurrences can be considered recrudescence. From 29 days (or even earlier in the case of resistant parasites) up to six months, recurrences can be considered relapses in the case of the tropical strain. Meanwhile, reinfections can occur at any moment after elimination of the drug from the system 2,13.

The Brazilian National Malaria Control Program (PNCM) conducts systematic investigation of recurrences by monitoring parasitemia at various intervals after the conclusion of chemotherapy. The PNCM defines recrudescence, probable relapse, and reinfection with $P$. vivax as the resurgence of parasitemia at intervals of 7 to 28 days, 29-60 days, and greater than 60 days, respectively, after initiating treatment of the primary infection. These cutoff points are arbitrary and can result in erroneous patient classification 14 .

Due to the operational difficulty in distinguishing relapses from reinfections, the current study aimed to describe and analyze all recurrences of P. vivax malaria in an endemic municipality (county) in the Brazilian Amazon, in order to better understand the phenomenon of repeated illness in the population due to this parasitic infection and to promote discussion of its impact on the prevailing control strategy.

\section{Methodology}

This was an open historical cohort study constructed from secondary data on malaria and its recurrences from January to December 2009 in the Municipality of Porto Velho, Rondônia, Brazil. The data were obtained from the National Information System on Malaria Epidemiological Surveillance (SIVEP-Malaria). The databank was first organized in Excel (Microsoft Corp., USA) and classified in alphabetical order by patient's name, mother's name, date of birth, and date of notification to ensure identification of recurrences. Duplicate notification was defined as the entry of data for the same patient on the same date of diagnosis or treatment for malaria, and was then excluded from the databank. Recurrent cases were defined as patients present in the databank with different diagnostic and treatment dates and which showed agreement between the patient's name, mother's name, and date of birth. Only those which showed agreement between 
at least three of these four variables were classified as recurrent cases. This stage of the study was completely manual and conducted entirely by the study's principal investigator.

For the analysis proposed in the study, the data were subsequently exported to Epidata Analysis, version 2.2.1.171 (Epidata Assoc., Odense, Denmark) and Stata, version 6.0 (Stata Corp., College Station, USA). Stratification of recurrent cases into different types used the cutoff points for time elapsed since last malaria treatment, according to PNCM guidelines 9 . Incidence of recurrences and respective $95 \%$ confidence intervals (95\%CI) during the target year were analyzed in relation to demographic (gender, age, and schooling) and clinical variables (time since onset of symptoms and parasitemia level in the primary infection), drawing this available data from SIVEP-Malaria. Determination of individual exposure time to the target event was based on the number of days elapsed since the first episode of the disease in 2009 and the first recurrence. The sum of these times calculated for each individual represented the person-time sampling universe, used to calculate the incidence density of recurrences. Thus, the time counted for patients that presented recurrent malaria during the year referred only to the date on which the patient entered the system for the first time, i.e., at the first malaria episode during the study year and the number of times they returned to the system.

Statistical analysis began with estimation of simple frequencies for the target variables. Demographic, clinical, and laboratory factors associated with incidence density of recurrences were identified by univariate analysis, determining relative risk (RR) and respective 95\%CI. Proportions between groups were compared using Pearson chi-square $\left(\chi^{2}\right)$ or Z-score. Means were compared using the Student $t$-test with the respective descriptive levels ( $p$-value). The effect of the accumulated number of repeated recurrences in the same individual during the time elapsed between each episode of recurrence was analyzed by simple linear regression. Significance was set at $5 \%$ for all the conclusions on the analysis of factors associated with recurrence.

A comparative evaluation of the incidence densities of recurrences between groups used survival analysis, constructing overall and group survival function curves with the Kaplan-Meier product estimator. In this analysis the accumulated risk of an individual presenting a recurrence over time after treatment of the initial symptomatic infection assumed independence of the event's occurrence between the different moments in time. Comparison of the survival curves without recurrence, constructed for the subgroups, used the Mantel-Haenszel log-rank test. Finally, the Cox proportional risks model was used to estimate the effect of the study's covariates on time until recurrence.

Access to the SIVEP was authorized by the PNCM, respecting non-disclosure of the patients' identity. The study was approved by the Ethics Research Committee at the Júlio Muller University Hospital, Federal University of Mato Grosso (case review 600/CEP/HUJM/09, March 18, 2009).

\section{Results}

During the year 2009, 26,296 patients with malaria were notified in the Municipality of Porto Velho. Analysis of the information recorded in SIVEP-Malaria showed duplicate notification of 290 patients, which were excluded from the database constructed in this study. Thus, 26,006 patients were analyzed, of whom 23,365 (89.8\%) were treated for malaria caused by P. vivax, 2,580 (9.9\%) for P. falciparum, and $61(0.2 \%)$ for mixed infection, that is, simultaneous infection with $P$. vivax and $P$. falciparum. The majority were males $(64.0 \%)$, young adults from 20 to 49 years of ages $(57.4 \%)$, with low schooling $(79.2 \%)$, and living in the Municipality of Porto Velho (94.1\%). Malaria transmission was relatively stable in 2009, with a slight increase in P. vivax cases in July and a drop from August to December (Figure 1). The chemotherapy regimen based on chloroquine + primaquine, recommended by the Brazilian Ministry of Health, was used to treat all patients with $P$ vivax malaria.

Of the 26,006 patients included in study, 5,958 (22.9\%) presented recurrences, of which 5,363 (90\%) were caused by P. vivax; 572 (9.6\%) by P.falciparum; and $23(0.4 \%)$ by mixed infection. During the year 2009, multiple recurrences (as many as eleven) were observed in various patients and for both Plasmodium species. For P. vivax, recurrences occurred as follows: one only in $67 \%$ of the patients, two in $21.5 \%$, three in $7.4 \%$, and four or more in $4.1 \%$. The most frequent type of recurrence in 2009 was probable reinfection with P. vivax, representing $65.3 \%$ of the recurrent cases. Probable relapses occurred in 1,760 patients (29.5\%) and recrudescence in 279 (5.2\%) (Table 1).

P. vivax recurrences were proportionally more frequent in males $(64.7 \%)$, in individuals from 12 to 49 years of age $(73.1 \%)$, and in those with low schooling (88.9\%). Mean age of patients with recurrence was 26.8 years (SD: 15.7). The most frequent level of parasitemia at diagnosis was 100 to 500 parasites $/ \mathrm{mm}^{3}$. Among the 1,018 childbear- 


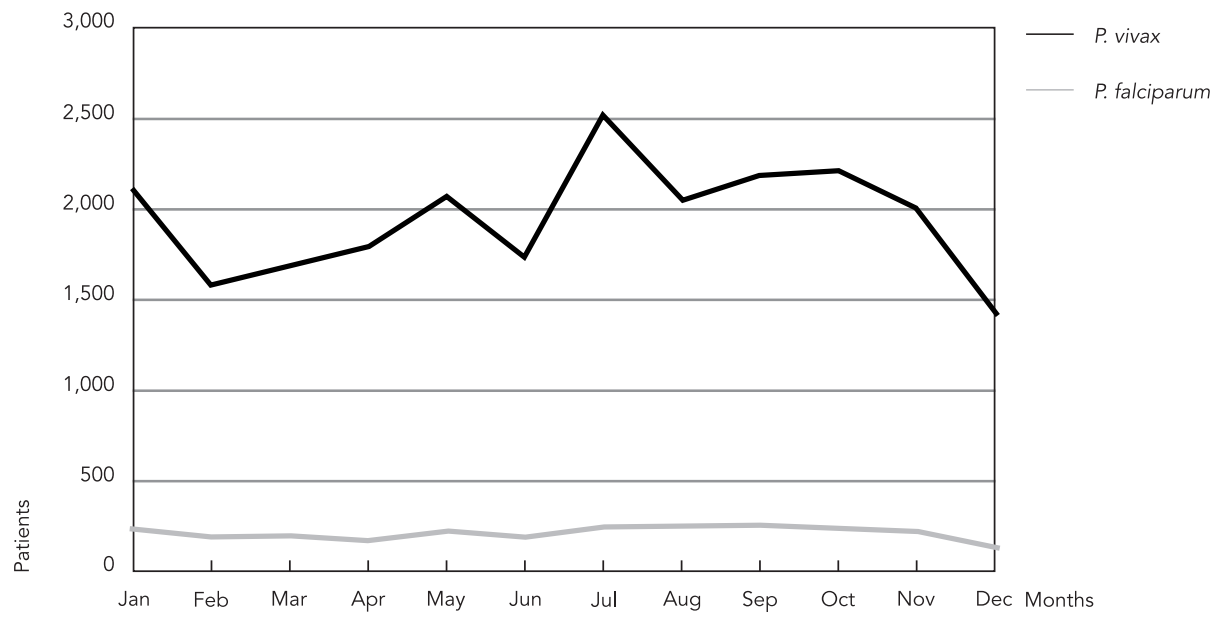

ing-age women analyzed, 215 were pregnant, of whom 26 (12.1\%) presented recurrent $P$. vivax infection. $P$. falciparum was the causative species of previous infection in only $6.6 \%$ of the patients that presented recurrence (Table 2).

Analysis of the time elapsed until the first recurrence with $P$. vivax in Porto Velho (2009) showed an asymmetrical shift to the right in the distribution, with a mean of 87.5 days (SD: 59.0), median of 69.5 (Q1-Q3: 48-106), and mode of 34 days. This distribution differed from that observed in recurrent $P$. falciparum malaria, which also showed a shift to the right, but with a median of 42 days and mode of 14 days (Figure 2). The difference in the intervals between each recurrence was progressively smaller $[\beta=-7.57$; $95 \% \mathrm{CI}$ : -9.19 ; -5.95; $\mathrm{p}<0.0001$ ] as the number of repetitions in recurrences per patient increased over the course of the year (Figure 3).

The 23,365 patients in the study had their first symptomatic episode of $P$. vivax infection in different months of 2009, resulting in variation in individual follow-up time in this historical cohort until occurrence of the target outcome, namely recurrence. Total follow-up time was thus $2,908,181$ person-days, or 7,967.2 person-years. A total of 3,595 patients experienced at least one $P$. vivax recurrence in 2009 . This resulted in an incidence density of $45.1 / 100$ person-years (95\%CI: 44.4; 45.3)/100.
Incidence of first recurrence was higher in men (RR = 1.10; 95\%CI: 1.02; 1.17; $\mathrm{p}=0.009)$ and was only associated with age greater than 50 years. Recurrence rate was also associated with level of education in patients with four to seven years of schooling ( $p=0.002)$. However, no association was observed with level of schooling less than three years $(p=0.214)$ or eight years or more ( $p=0.897$ ). Incidence of first recurrence in pregnant and non-pregnant women was similar $(\mathrm{RR}=0.91 ;$ 95\%CI: 0.62; 1.34; $\mathrm{p}=0.641)$. Patients' clinical characteristics showed that first recurrence was less frequent in patients with more than 72 hours since onset of symptoms and more frequent in those with parasitemia greater than $10,000 / \mathrm{mm}^{3}$ at the first symptomatic infection in 2009 (Table 3).

After adjusting the probability of recurrence over time, the following remained significantly associated with the outcome: male gender $(\mathrm{HR}=1.10 ; 95 \% \mathrm{CI}: 1.03 ; 1.18 ; \mathrm{p}=0.006)$, time since onset of symptoms 48-72 hours ( $\mathrm{HR}=1.21$; 95\%CI: $1.08 ; 1.35 ; \mathrm{p}=0.001$ ) or less than 48 hours (HR $=1.36$; 95\%CI: 1.25; 1.49; $<<0.0001$ ), and having peak parasitemia at diagnosis of 501-10,000/ $\mathrm{mm}^{3}$ (HR = 1.32; 95\%CI: 1.23; 1.43; $\left.\mathrm{p}<0.0001\right)$ or greater than $10,000 / \mathrm{mm}^{3}(\mathrm{HR}=1.44 ; 95 \% \mathrm{CI}$ : 1.19; 1.75; $\mathrm{p}<0.0001$ ) (Table 4; Figure 4). 
General characteristics of recurrent Plasmodium vivax malaria in the Municipality of Porto Velho, Rondônia State, Brazil, 2009.

\begin{tabular}{lcc}
\hline Characteristics & $\mathbf{n}$ & $\%$ \\
\hline Recurrences $(\mathrm{n}=23,365)$ & & 23.0 \\
Yes & $5,363 *$ & 77.0 \\
No & 18,002 & \\
Number of recurrences per patient in 2009 & 3,595 & 67.0 \\
One & 1,154 & 21.5 \\
Two & 396 & 7.4 \\
Three & 148 & 2.8 \\
Four & 71 & 1.8 \\
Five or more & & \\
Type of recurrence ** & 186 & 5.2 \\
Recrudescence $(<28$ days $)$ & 1,061 & 29.5 \\
Probable relapse $(28-60$ days $)$ & 2,348 & 65.3 \\
Probable reinfection $(>60$ days) & & \\
\hline
\end{tabular}

* Some patients had more than one recurrence during the study period;

** Classification based on the guidelines of the Brazilian National Malaria Control Program, Ministry of Health.

\section{Discussion}

The recurrence rate for P. vivax infection in Porto Velho was high in 2009. The initial hypothesis for these recurrences was reactivation of hypnozoites, which are not always eliminated by primaquine, due either to resistance to this drug or another factor that interferes with its action 15 . From the epidemiological point of view, little has been published on the magnitude of recurrences after treatment of $P$. vivax malaria in a given endemic region, based on the interpretation of this phenomenon as the combination of recrudescence, relapse, and reinfection, as defined above. However, the proportion of $P$. vivax infections that evolve to relapses has been well-studied, and it is known to be an intrinsic property of the malaria parasite. The proportion varies considerably between geographic regions and is higher with the species' tropical strain when compared to the temperate strain. It is also a function of the size of the sporozoite inoculum and the patient's immunity, when the latter exists $2,15,16$. The probability of relapse with a single genotype strain is also known to depend on the time since the patient has been ill, which also suggests the effect of immunity on the occurrence of this phenomenon 2 .

The high proportion of malaria cases caused by $P$. vivax as compared to $P$. falciparum in Porto Velho was no different from that recorded in the Amazon Region as a whole that same year. The same was true for the predominance in males, young adults, and individuals with low school- ing 2. The $23.0 \%$ overall recurrence for $P$. vivax malaria in this municipality in the year 2009 can be considered high, although not very different from the levels for the Legal Amazonia during the same period, namely $20.8 \%$, identified as LVC (lâmina de verificação de cura, or cure verification smear) in the SIVEP-Malaria database ${ }^{1}$. It was also similar to the rates observed by Prasad et al. 15 in a study of reinfection/relapse in India (23.2\%), Adak et al. 17 in a study of $P$. vivax recurrence patterns, also in India (23.3\%), and Haghdoost et al. 18 in a study in Iran (16.8\%), also in one-year population follow-up.

In Brazil, a retrospective study of cases of symptomatic $P$. vivax infection reported in São Paulo in the 1980s (when malaria was still autochthonous in that state) showed a similar recurrence rate after treatment of symptomatic $P$. vivax infection $(24.5 \%)$ 19. Meanwhile, in a study in a nonendemic area without risk of transmission, $P$. vivax recurrence (observed as relapse after treatment with primaquine) occurred less frequently (14\%) than in the current study, probably due to treatment supervision ${ }^{3}$.

Overall incidence of $P$. vivax recurrence in Porto Velho (2009) was 45.1/100 person-years. The high rate expresses the risk of an individual with symptomatic $P$. vivax infection in that municipality presenting a new episode of malaria from the same species over the course of a year. In countries like Thailand and Papua New Guinea, the P. vivax recurrence rate after treatment with chloroquine and primaquine can reach $65 \%$ in 30 to 180 days 
Demographic and clinical characteristics of patients with at least one recurrence (R1) of Plasmodium vivax malaria. Municipality of Porto Velho, Rondônia State, Brazil, 2009.

\begin{tabular}{|c|c|c|}
\hline Characteristics & $\mathrm{n}$ & $\%$ \\
\hline \multicolumn{3}{|l|}{$\operatorname{Sex}(n=3,595)$} \\
\hline Male & 2,327 & 64.7 \\
\hline Female & 1,268 & 35.3 \\
\hline \multicolumn{3}{|l|}{ All females $(n=1,018)$} \\
\hline Pregnant & 26 & 2.6 \\
\hline Not pregnant & 992 & 97.4 \\
\hline \multicolumn{3}{|l|}{ Age (years) } \\
\hline Mean (SD) & \multicolumn{2}{|c|}{$26.8(15.7)$} \\
\hline Median (Q1-Q3) & \multicolumn{2}{|c|}{$25.0(14.0-38.0)$} \\
\hline \multicolumn{3}{|c|}{ Age bracket (years) $(n=3,595)$} \\
\hline$<12$ & 652 & 18.1 \\
\hline $12-19$ & 678 & 18.9 \\
\hline $20-49$ & 1,950 & 54.2 \\
\hline$>50$ & 315 & 8.8 \\
\hline \multicolumn{3}{|l|}{ Years of schooling $(n=3,376)$} \\
\hline None & 301 & 8.9 \\
\hline $1-3$ & 2,701 & 80.0 \\
\hline $4-7$ & 239 & 7.1 \\
\hline $8-11$ & 135 & 4.0 \\
\hline \multicolumn{3}{|c|}{ Species of infection prior to the recurrence $(n=3,595)$} \\
\hline Plasmodium falciparum & 238 & 6.6 \\
\hline Plasmodium vivax & 3,357 & 93.4 \\
\hline \multicolumn{3}{|c|}{ Parasitemia at first episode/mm3 $(n=3,595)$} \\
\hline $100-500$ & 2,059 & 57.3 \\
\hline $501-10,000$ & 1,393 & 38.7 \\
\hline$>10,000$ & 143 & 4.0 \\
\hline \multicolumn{3}{|c|}{ Time since onset of symptoms (hours) $(n=3,079$ ) } \\
\hline$<48$ & 1,741 & 56.5 \\
\hline $48-72$ & 581 & 18.9 \\
\hline$>72$ & 757 & 24.6 \\
\hline
\end{tabular}

Note: variation due to missing information for the variable.

of follow-up 20,21. However, the rate had not been studied in the endemic region of the Brazilian Amazon. The few available studies were conducted in an area without transmission ${ }^{3}$ and in the endemic area of the Atlantic Forest, where the risk of new infections is very low ${ }^{19}$. A recent study by OrjuelaSanchez et al. ${ }^{9}$ evaluated P. vivax incidence and time to recurrence in two cohorts of patients living on the Brazil-Peru-Bolivia border, treated with chloroquine + primaquine. The authors found 180 -day accumulated incidence rates of $26 \%$ and $40 \%$ for $P$. vivax recurrence in these two patient cohorts.

The following factors may explain the high recurrence rate in the current study. The first was the high level of $P$. vivax transmission in Porto Velho in 2009, favoring new infections. During that year, malaria incidence in Porto Velho (as recorded in the SIVEP-Malaria database) exceeded 50 cases/1,000 inhabitants, characterizing high transmission risk in the municipality ${ }^{1}$. The second was the high recrudescence/relapse rate, due to possible treatment failures with the chloroquine + primaquine regimen recommended by the PNCM for treatment of this species throughout the Amazon ${ }^{3}$. Since malaria treatment is not supervised in the Amazon Region and the antimalarial dispensing system in health services is often precarious 2 , this hypothesis cannot be overlooked. Neither is it possible to rule out factors associated with the total 
Distribution of time elapsed between initial episode and first recurrence of Plasmodium vivax (2a) and Plasmodium falciparum malaria (2b). Municipality of Porto Velho, Rondônia State, Brazil, 2009.

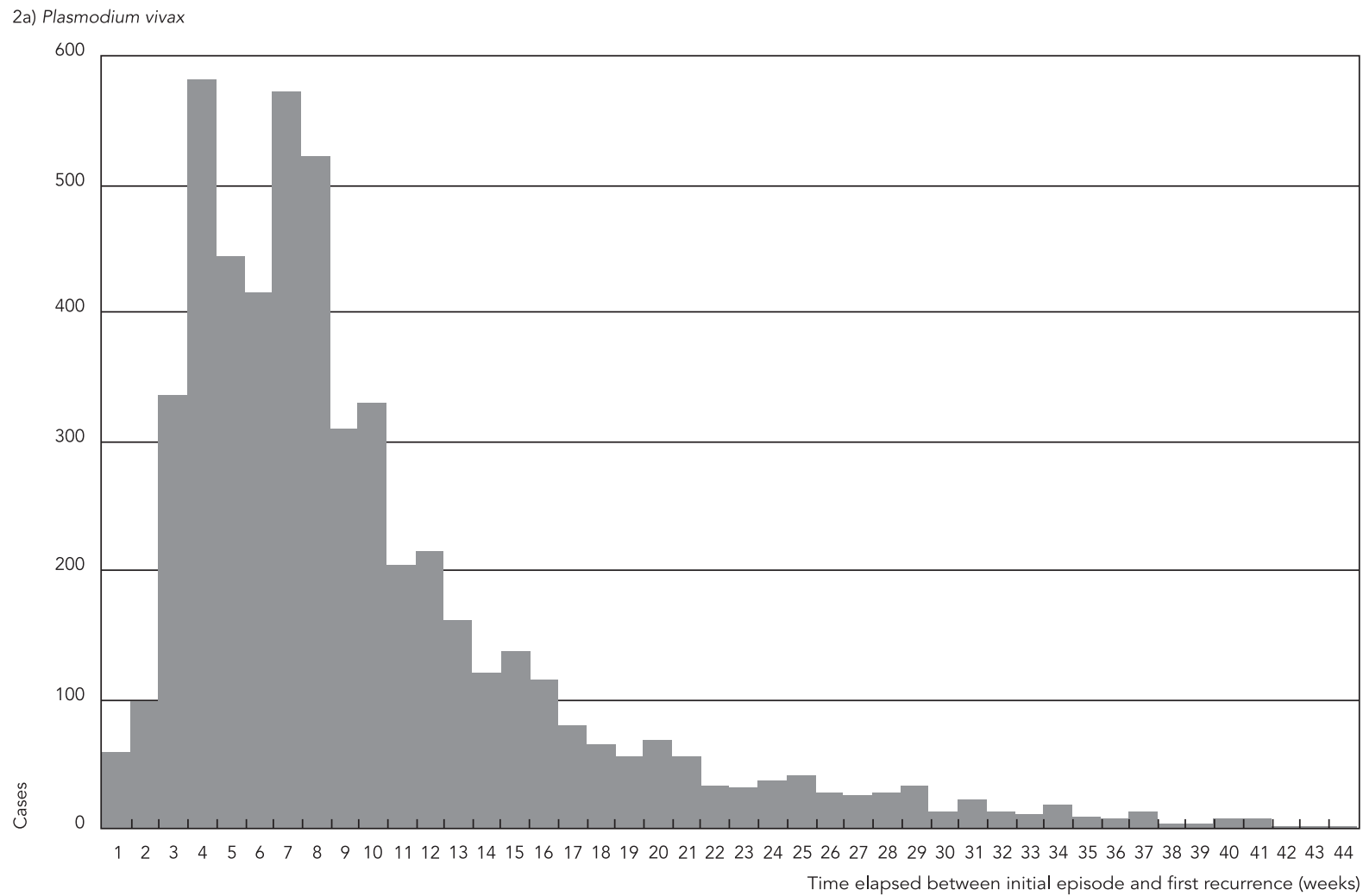

(continues)

administered dose of primaquine and the development of hypnozoite resistance to this drug in the region, as other researchers have suggested 3,22 . Another issue that cannot be overlooked is Brazil's recommendation of the short (7-day) primaquine regimen, unlike the 14-day regimen recommended in most endemic regions of the world 3,4.

The number of recurrences in patients with recurrent $P$. vivax malaria in 2009 in Porto Velho varied from 1 to 11 . This high rate of recurrent malaria in such a short time interval had already been reported previously, as the exclusive result of hypnozoite reactivation 2 . During World War II, $70 \%$ to $80 \%$ of American soldiers stationed in Southeast Asia experienced relapses of P. vivax malaria (tropical strain) after returning to the United States, many of whom (9\%) more than ten times in a year 23. Other authors also observed the predominance of recurrences (and in varying numbers) within the first year after treatment of the primary infection, despite correct primaquine dosage ${ }^{3}$. In areas where the long and short latency strains are known to occur simultaneously, as in India, radical treatment of $P$. vivax is more effective for the temperate strain than the tropical strain 2 . These findings shed doubt on the efficacy of primaquine and raise hypotheses on possible phenotypic characteristics of $P$. vivax, making hypnozoites more resistant to eradication 24 .

Prospective studies in India in the last 25 years showed relapse rates varying from $8.6 \%$ to $40.1 \%$ following treatment of $P$. vivax exclusively with chloroquine (without combined primaquine) 17,25. It is suggested that without radical treatment with primaquine, a constant proportion of patients experience one, two, three, or more relapses, with an exponential ratio ${ }^{2}$. It is thus estimated that in areas with a relapse rate of $50 \%$ or more, approximately $6.25 \%$ of patients will have four or more relapses after a single symptomatic primary infection. This information is extremely relevant for $P$. vivax malaria control, since it provides an indication of the 
2b) Plasmodium falciparum

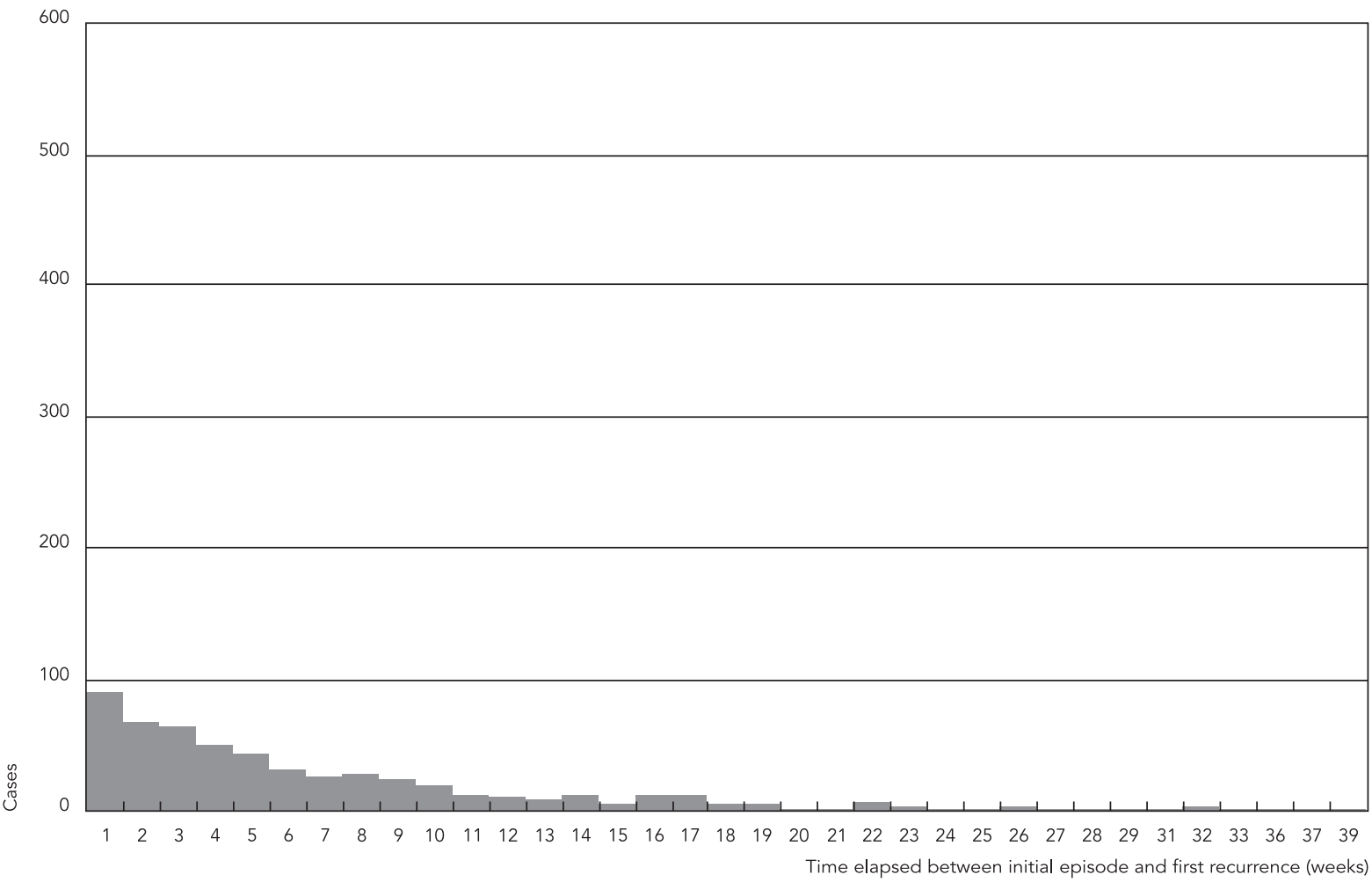

circulating hypnozoite burden in a given area. That is, if the relapse rate exceeds $50 \%$, the principal determining factor in the persistence of malaria in an area is hypnozoite reactivation in patients previously infected with P. vivax. This should therefore be the priority target for control measures 2 .

The monthly recurrence rate increased rapidly in the first three months of 2009, stabilizing around $22 \%$ in the subsequent months, without displaying a seasonal pattern. Studies in American soldiers showed that the first relapse occurred 60 days after the primary attack by P. vivax, recurring some 300 days later 2 . The low recurrence rate in the initial months of this cohort was expected, due to the definition of recurrence used in the study. Many of the malaria episodes during this period may actually have been recurrences of initial infections acquired in the previous year. In fact, analyzing only the period from April to December 2009, the monthly recurrence rate accompanied the malaria endemic curve in Porto
Velho during the same year, showing that recurrences in the current study also resulted from reinfections that occurred simultaneously with relapses of the symptomatic $P$. vivax infection.

The mean time elapsed between the first symptomatic infection in 2009 and first recurrence, as well as between the first subsequent recurrence and the previous treatment episode, varied and decreased with the increase in the frequency of recurrent episodes. Lysenko et al. 26 , reviewed by White ${ }^{2}$, also found a progressive reduction in the $P$. vivax relapse intervals, in both the temperate and tropical strains of the parasite. A plausible theory to explain variability in the intervals between relapses is that the sporozoites inoculated by the vector are actually a mixture of genetically distinct parasites that induced two populations of hypnozoites, i.e., with short and long latency 26.

The highest risk of recurrent P.vivax in a patient in 2009 occurred between the fourth and ninth 
Distribution of recurrences of Plasmodium vivax malaria according to mean time between episodes. Municipality of Porto Velho, Rondônia State, Brazil, 2009.

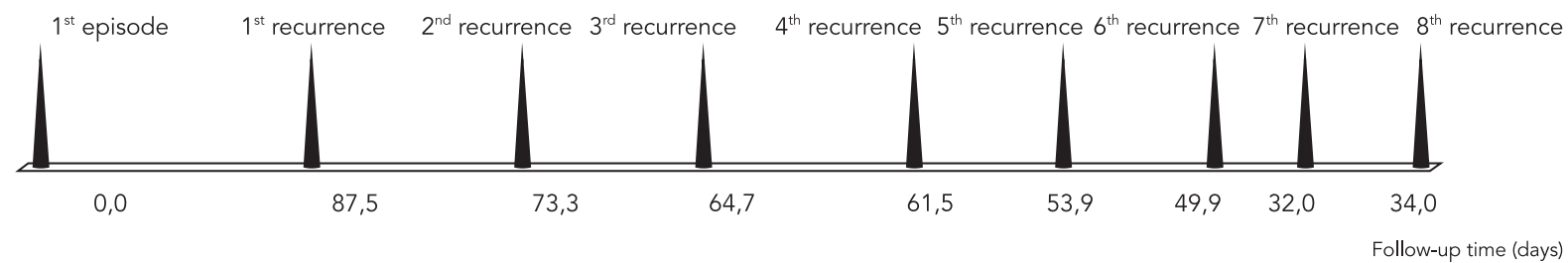

$\beta=-7.57 ; 95 \% \mathrm{Cl}:-9.19 ;-5.95(\mathrm{p}<0.0001)$

Table 3

Analysis of demographic and clinical factors associated with first recurrence of Plasmodium vivax malaria. Municipality of Porto Velho, Rondônia State, Brazil, 2009.

\begin{tabular}{|c|c|c|c|c|c|}
\hline \multirow[t]{2}{*}{ Characteristics } & \multicolumn{2}{|c|}{ Recurrence } & \multirow[t]{2}{*}{$\mathrm{RR}$} & \multirow[t]{2}{*}{$95 \% \mathrm{Cl}$} & \multirow[t]{2}{*}{ p-value * } \\
\hline & $\mathrm{n}$ & $\begin{array}{l}\text { ence density } \\
0 \text { person-years) }\end{array}$ & & & \\
\hline \multicolumn{6}{|l|}{ Sex } \\
\hline Female & 1,268 & 42.6 & 1.10 & $1.02 ; 1.17$ & 0.009 \\
\hline Male & 2,327 & 46.6 & & & \\
\hline \multicolumn{6}{|c|}{ Pregnant $(n=1,018)$} \\
\hline No & 992 & 41.9 & 0.91 & $0.62 ; 1.34$ & 0.641 \\
\hline Yes & 26 & 38.4 & & & \\
\hline \multicolumn{6}{|c|}{ Age bracket (years) } \\
\hline$<12$ & 652 & 45.8 & 1.00 & - & - \\
\hline $12-19$ & 678 & 47.1 & 1.03 & $0.93 ; 1.14$ & 0.620 \\
\hline $20-49$ & 1,950 & 46.1 & 1.01 & $0.92 ; 1.10$ & 0.888 \\
\hline$>50$ & 315 & 35.8 & 0.78 & $0.68 ; 0.89$ & 0.0003 \\
\hline \multicolumn{6}{|l|}{ Schooling (years) } \\
\hline None & 301 & 40.2 & 1.00 & - & - \\
\hline $1-3$ & 2,701 & 43.4 & 1.08 & $0.96 ; 1.21$ & 0.214 \\
\hline $4-7$ & 239 & 52.6 & 1.30 & $1.10 ; 1.55$ & 0.002 \\
\hline $8-11$ & 135 & 40.8 & 1.02 & $0.83 ; 1.24$ & 0.897 \\
\hline \multicolumn{6}{|c|}{ Onset of symptoms (hours) } \\
\hline$>72$ & 757 & 36.8 & 1.00 & - & - \\
\hline $48-72$ & 581 & 43.9 & 1.19 & $1.07 ; 1.33$ & 0.001 \\
\hline$<48$ & 1,741 & 49.4 & 1.35 & $1.24 ; 1.47$ & $<0.0001$ \\
\hline \multicolumn{6}{|c|}{ Parasitemia at first episode $\left(\mathrm{mm}^{3}\right)$} \\
\hline $100-500$ & 2,059 & 40.6 & 1.00 & - & - \\
\hline $501-10,000$ & 1,393 & 52.7 & 1.30 & $1.21 ; 1.39$ & $<0.0001$ \\
\hline$>10,000$ & 143 & 58.6 & 1.44 & $1.22 ; 1.71$ & $<0.0001$ \\
\hline
\end{tabular}

95\% Cl: 95\% confidence interval; RR: relative risk.

* Descriptive test level ( $p$-value) calculated by Z-score. 
Multivariate analysis of demographic and clinical factors associated with probability of first recurrence of Plasmodium vivax malaria. Municipality of Porto Velho, Rondônia State, Brazil, 2009

\begin{tabular}{|c|c|c|c|c|c|}
\hline \multirow[t]{2}{*}{ Characteristics } & \multicolumn{2}{|c|}{ First recurrence } & \multirow[t]{2}{*}{$\mathrm{HR}$} & \multirow[t]{2}{*}{$95 \% \mathrm{Cl}$} & \multirow[t]{2}{*}{ p-value * } \\
\hline & $\mathbf{n}$ & $\%$ & & & \\
\hline \multicolumn{6}{|l|}{ Sex } \\
\hline Female & 1,268 & 19.4 & 1.10 & $1.03 ; 1.18$ & 0.006 \\
\hline Male & 2,327 & 20.8 & & & \\
\hline \multicolumn{6}{|c|}{ Age bracket (years) } \\
\hline $0-12$ & 652 & 20.6 & 1.00 & - & - \\
\hline $12-19$ & 678 & 21.5 & 1.18 & $1.01 ; 1.37$ & 0.036 \\
\hline $20-49$ & 1,951 & 20.5 & 1.31 & $1.13 ; 1.53$ & $<0.0001$ \\
\hline$>50$ & 315 & 16.6 & 1.27 & $1.12 ; 1.46$ & $<0.0001$ \\
\hline \multicolumn{6}{|l|}{ Schooling (years) } \\
\hline None & 301 & 19.0 & 1.00 & - & - \\
\hline $1-3$ & 2,701 & 20.2 & 1.05 & $0.91 ; 1.20$ & 0.501 \\
\hline $4-7$ & 239 & 22.3 & 1.13 & $0.93 ; 1.37$ & 0.211 \\
\hline $8-11$ & 135 & 19.8 & 0.95 & $0.76 ; 1.19$ & 0.640 \\
\hline \multicolumn{6}{|c|}{ Onset of symptoms (hours) } \\
\hline$>72$ & 757 & 24.6 & 1.00 & - & - \\
\hline $48-72$ & 581 & 18.9 & 1.21 & $1.08 ; 1.35$ & 0.001 \\
\hline$<48$ & 1,741 & 56.5 & 1.36 & $1.25 ; 1.49$ & $<0.0001$ \\
\hline \multicolumn{6}{|c|}{ Parasitemia (mm3) } \\
\hline $100-500$ & 2,059 & 57.3 & 1.00 & - & - \\
\hline $501-10,000$ & 1,393 & 38.8 & 1.32 & $1.23 ; 1.43$ & $<0.0001$ \\
\hline$>10,000$ & 143 & 3.98 & 1.44 & $1.19 ; 1.74$ & $<0.0001$ \\
\hline
\end{tabular}

HR: hazard ratio; 95\% Cl: 95\% confidence interval.

* Cox regression

week after treatment of the first malaria. Since low adherence to malaria treatment it not that common in the Brazilian Amazon 2, these early recurrences may actually result from relapses due to hypnozoite activation. The so-called high relapse frequency strains of $P$. vivax (including the tropical strain) have already been recorded in South America, India, Southeast Asia, and Oceania. However, the strains with long latency hypnozoites also occurred in these same areas ${ }^{2}$. This overlapping could thus explain the wide range in time to recurrence observed in the current study, i.e., the early recurrences caused by relapses of tropical strains and the later ones by relapses of long latency strains. In Brazil, the predominant situation is presumed to be that the tropical strain of P. vivax, characterized by early primary attack, is followed by a short latency period until the relapses that appear during the subsequent year. However, no scientific evidence is available on this issue in Brazil or in the rest of South America. Researchers in Rio de Janeiro and Brasília have recently reported travelers with long incubation periods for malaria, varying from 3 to 12 months after returning from trips to the Amazon. They suggested that temperate strains coexist with the tropical strain in the country 22,27 . This hypothesis is corroborated by the observation of relapses at days $33,73,82,88$, 113,121 , and 137 after initiating therapy in a $P$. vivax malaria treatment cohort in 1990 in Cuiabá (Mato Grosso State), where malaria transmission does not occur ${ }^{3}$.

Based on the operational classification of recurrences proposed by the PNCM, $29.5 \%$ of P. vivax recurrences in Porto Velho in 2009 were probable relapses. It is quite probable that this estimated proportion is close to the true proportion of relapses in the study population, because the reappearance of $P$. vivax in relapses after a primary infection occurred between 28 days (3\%) and 63 days (63\%) in a study in Thailand 28 . Furthermore, $P$. vivax recurrence was observed between 28 and 90 days in $70 \%$ of patients studied in French Guiana, although recrudescence and 
Kaplan-Meier curves for probability of remaining without recurrence of malaria, according to age bracket (4a), time since onset of symptoms (4b), and level of parasitemia (4c). Municipality of Porto Velho, Rondônia State, Brazil, 2009.

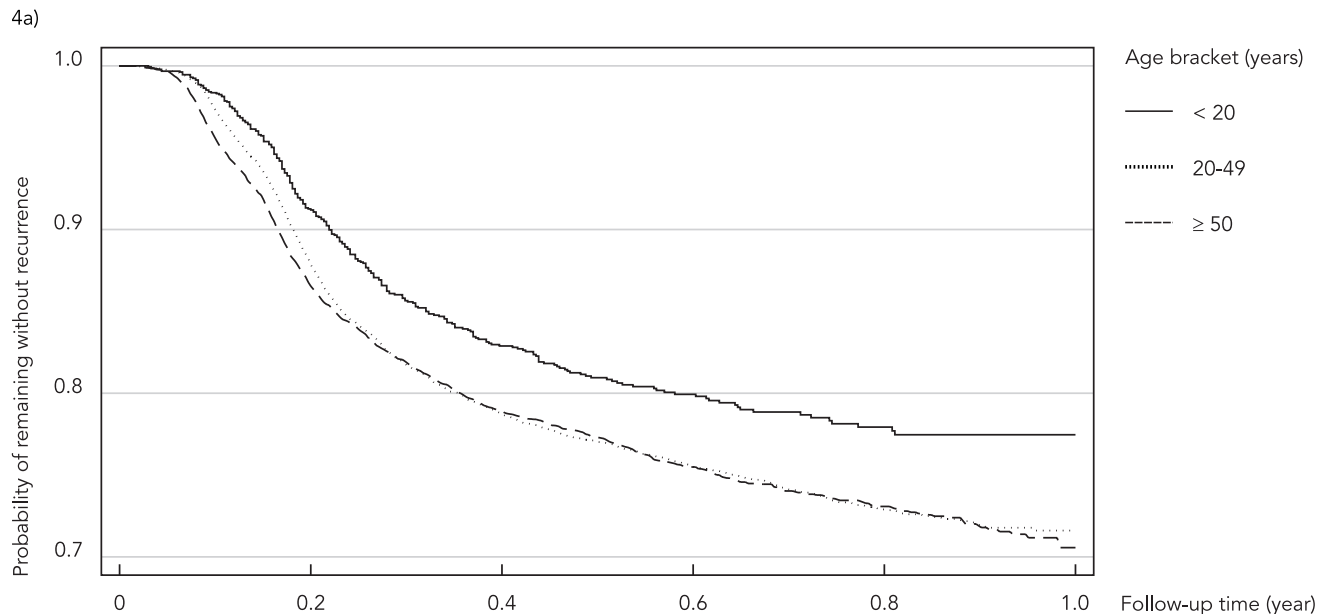

4b)

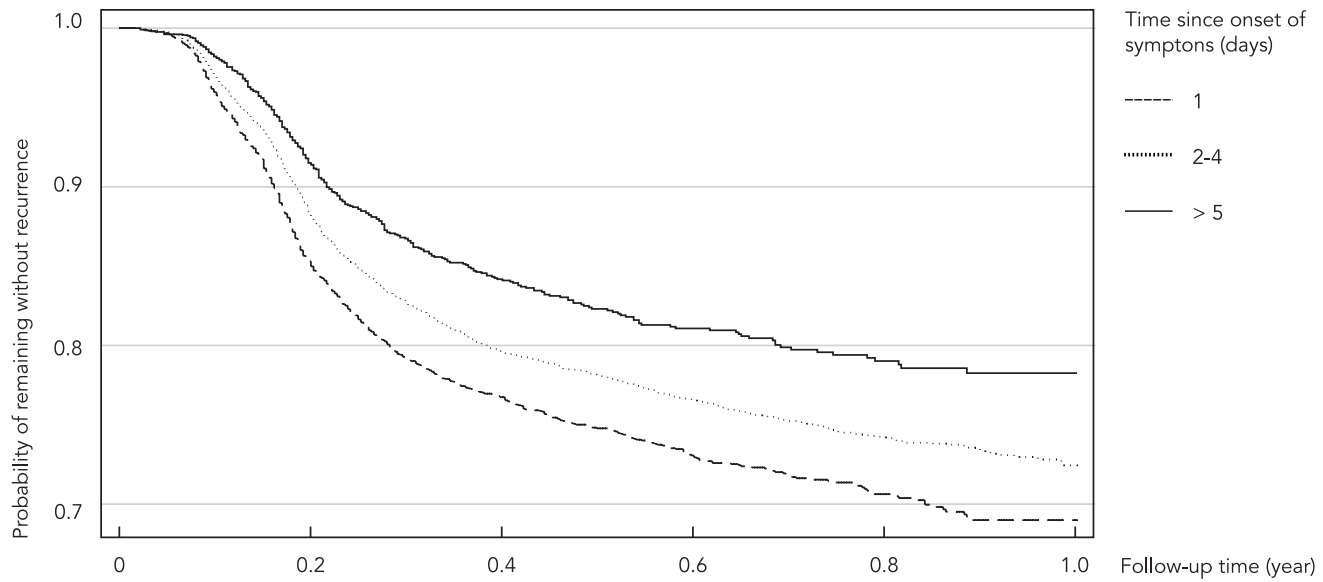

4c)

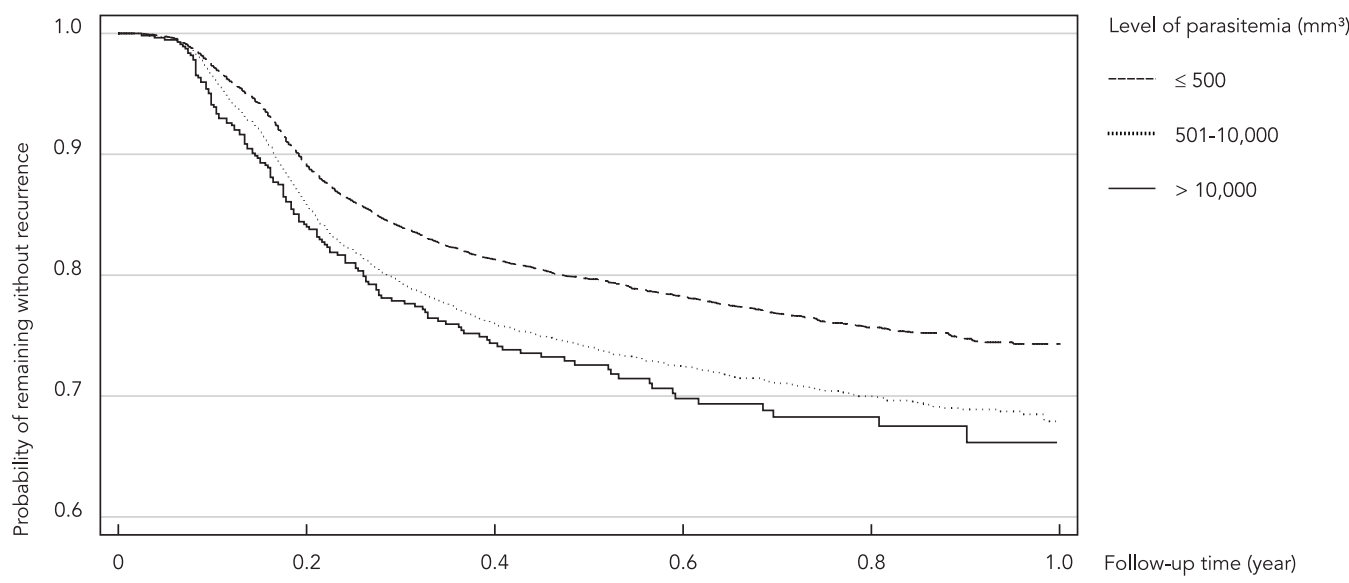


reinfections could not be ruled out, as occurred in the present study 29 .

A higher recurrence rate was expected among pregnant women. The risk of relapses from $P$. vivax malaria in individuals that do not receive primaquine is known to range from $5 \%$ to $80 \%$, depending on the geographic region 30 . A likely explanation for the low recurrence rate observed in this group is the use of hypnozoite suppressive therapy with weekly chloroquine $(5 \mathrm{mg} / \mathrm{kg})$, recommended since 2008 by the PNCM for patients in whom primaquine is contraindicated 14 .

Although some studies have not found a higher recurrence rate in men 18 , this finding was observed by Prasad et al. 15, who suggested low treatment adherence as the probable explanation for this difference, highlighting greater risk of treatment dropout by men due to low schooling and longer workweeks (as contributing factors). Thus, recurrences in women would tend to be due more to relapses or reinfections. In addition to relapses and reinfections, men would also show a higher recrudescence rate due to low treatment adherence.

No age bracket in the patients studied here was associated with greater risk of recurrences in Porto Velho (2009). In endemic areas in Thailand, the age profile of patients with $P$. vivax malaria suggests more rapid and intense acquisition of immunity with this species than with $P$. falciparum 30 . Various studies on this issue have suggested that both the proportion of $P$. vivax relapses and the rate of entomological inoculation decline in elderly individuals. Immunity and thus age are probable confounders in epidemiological evaluations of recurrence and relapse from $P$. vivax malaria 2,31 .

The recurrence rate for $P$. vivax malaria was low following symptomatic infection with $P$. falciparum. In Southeast Asia, the incidence of this phenomenon is substantially greater than expected according to the entomological inoculation rate, i.e., with no relationship to previous infection with a different species 32. In Ethiopia, where the early relapses rates for P. vivax malaria are lower, as in the current study, the phenomenon occurred in only $7 \%$ of patients 33 . Since the two species coexist in Porto Velho, recurrences from $P$. vivax following infections with $P$. falciparum probably result merely from the greater risk of transmission of the first species, as recorded in recent years.

Higher parasitemia in the first infection with $P$. vivax diagnosed in 2009 was associated with greater risk of recurrence. Recurrence also occurred earlier in patients with hyperparasitemia. In hyperendemic areas, higher parasite density for any Plasmodium species is seen in children, pregnant women, and adults with primary infec- tion, due to the lower level of naturally acquired immunity in these groups 34 . Therefore, the observed association is actually presumed to be the effect of lower immunity in patients with hyperparasitemia and consequently the effect on recurrence rate, as described for P. vivax relapses 2 .

Meanwhile, the longer the time since onset of symptoms (as reported by patients at time of diagnosis), the lower the rate of $P$. vivax recurrences, which also occurred later. One cause of longer time since onset of symptoms that cannot be ruled out is delay in diagnosis resulting from difficult access to health services, especially distance from the patient's home to the laboratory. However, the patients with the longest delays in seeking malaria diagnosis are probably the least symptomatic, having already developed some degree of clinical immunity, already described for malaria in individuals from endemic areas, causing a negative impact on the incidence of recurrence 2 . The current study also corroborates this with the lower recurrence rate among patients in higher age brackets, who are more likely to have naturally acquired immunity to malaria 35 .

In short, the study showed that male gender, shorter symptom time, and higher parasitemia at diagnosis of $P$. vivax malaria increased the risk of recurrence over the course of a year. The strength of association for each of these risk factors after the adjusted analysis included $11 \%$ and $15 \%$ increases in relative risk for males and patients with higher parasitemia, respectively, and a $10 \%$ reduction in risk of recurrence in patients with longer symptom time. Although these values for increased and decreased risk are not that large, they could help orient malaria control programs in choosing groups of patients to focus greater attention, in order to minimize their suffering from recurrences.

It is important to highlight some inherent limitations to this type of epidemiological study. Since the data were obtained from a secondary source, the results are not exempt from a possible selection bias due to underreporting of cases in the SIVEP-Malaria base. But this bias is likely to have been minimal, since the SIVEP-Malaria base is known as a sensitive and timely information system with good completeness. Another important issue that can underestimate the recurrence rate in the study is the patient's mobility in the endemic area, and the same patient may have had one or more recurrences diagnosed in a neighboring municipality or state. Although this possible loss was not measured, if it does exist, its influence on the study's result must not have interfered in the objective, since the observed recurrence rate was already high.

The low values for the risk measures in the multivariate analyses can be interpreted as hav- 
ing low clinical relevance for the observed associations, limiting their causal potential for predicting recurrence rates or justifying decisions at the public health level. Although further cohort studies including information on patients' clinical and behavioral patterns are necessary to better evaluate the real factors related to recurrent malaria, the current study's results are relevant for calling the attention of the PNCM to the high rate of recurrences from $P$. vivax malaria in Brazil and for reflecting on more effective control measures.

\section{Resumen}

Estudio de cohorte histórica abierta, construida con información registrada en el Sistema de Información de Vigilancia Epidemiológica de la Malaria, con el objetivo de describir la incidencia y los factores asociados a la reaparición de malaria, causada por el Plasmodium vivax, en Porto Velho, Rondônia, Brasil. Se notificaron 23.365 casos de malaria por P. vivax en el municipio, donde un $23 \%$ de ellos presentaron recidiva a lo largo del 2009. La densidad de incidencia de recidivas fue de 45,1/100 pacientes-año, produciéndose, principalmente, entre la $4^{a} y$ la $13^{a}$ semana tras el inicio del tratamiento. Ser hombre, tener menos tiempo síntomas y tener un mayor nivel de parasitemia en la infección inicial aumentaron el riesgo de recidiva a lo largo del año, con un 10\% de reducción relativa del riesgo, para un mayor tiempo de síntomas, y de un 11\% y 15\% de incremento relativo del riesgo para el sexo masculino y mayores niveles de parasitemia, respectivamente. No obstante, los resultados demostraron baja relevancia clínica de las asociaciones encontradas, comprometiendo su aplicabilidad en la toma de decisiones dentro de la salud pública.

Recurrencia; Plasmodium vivax; Malaria

\section{Contributors}

C. J. F. Fontes participated in the study design, elaboration of the research project, data analysis, and writing of the manuscript. L. R. Simões participated in the data organization and analysis and writing of the manuscript. E. R. Alves-Jr participated in the organization of the databank and critical revision of the manuscript. D. Ribatski-Silva participated in the data analysis and writing of the manuscript. L. T. Gomes participated in the data analysis and critical revision of the manuscript. A. F. Nery verified and conducted the final revision of the databank and critical revision of the manuscript.

\section{Acknowledgments}

The authors with to thank CNPq (case 555652/2009-2) and the FAPEMAT, PRONEX-Malaria Network, Mato Grosso, for the financial support. 


\section{References}

1. Secretaria de Vigilância Epidemiológica, Ministério da Saúde. Malária. Resumo epidemiológico por local de notificação. http://www.saude.gov.br/svs (accessed on 09/Aug/2013).

2. White NJ. Determinants of relapse periodicity in Plasmodium vivax malaria. Malar J 2011; 10:297.

3. Pereira EA, Ishikawa EAY, Fontes CJF. Adherence to Plasmodium vivax malaria treatment in the Brazilian Amazon Region. Malar J 2011; 10:355.

4. Duarte EC, Pang LW, Ribeiro LC, Fontes CJF. Association of subtherapeutic dosages of a standard drug regimen with failures in preventing relapses of vivax malaria. Am J Trop Med Hyg 2001; 65: 471-6.

5. Nogueira FH, Moreira-Campos LM, Santos RL, Pianetti GA. Quality of essential drugs in tropical countries: evaluation of antimalarial drugs in the Brazilian Health System. Rev Soc Bras Med Trop 2011; 44:582-6.

6. Adhikari P. Recurrent malaria: an enigma? Indian J Med Sci 2000; 54:325-9.

7. Imwong $M$, Snounou G, Pukrittayakamee S, Tanomsing N, Kim JR, Nandy A, et al. Relapses of Plasmodium vivax infection usually result from activation of heterologous hypnozoites. J Infect Dis 2007; 195:927-33.

8. Chen N, Auliff A, Rieckmann K, Gatton M, Cheng Q. Relapses of Plasmodium vivax infection result from clonal hypnozoites activated at predetermined intervals. J Infect Dis 2007; 195:934-41.

9. Orjuela-Sanchez P, Silva NS, Silva-Nunes M, Ferreira UM. Recurrent parasitemias and population dynamics of Plasmodium vivax polymorphisms in rural Amazonia. Am J Trop Med Hyg 2009; 81: 961-8.

10. Restrepo E, Imwong M, Rojas W, Carmona-Fonseca J, Maestre A. High genetic polymorphism of relapsing Plasmodium vivax isolates in northwest Colombia. Acta Trop 2011; 119:23-9.

11. Araujo F, Resende A, Fontes CJ, Carvalho L, Brito C. Multiple-clone activation of hypnozoites is the leading cause of relapse in Plasmodium vivax infection. PLoS One 2012; 7:e49871.

12. Markus MB. The hypnozoite concept, with particular reference to malaria. Parasitol Res 2011; 108:247-52.

13. Hanf M, Stephani A, Basurko C, Nacher M, Carme B. Determination of the Plasmodium vivax relapse pattern in Camopi, French Guiana. Malar J 2009; 8:278.

14. Secretaria de Vigilância Epidemiológica, Ministério da Saúde. Guia de vigilância epidemiológica. 7a Ed. Brasília: Ministério da Saúde; 2009.

15. Prasad R, Virk K, Sharma V. Relapse/reinfection patterns of Plasmodium vivax infection: a four year study. Southeast Asian J Trop Med Public Health 1991; 22:499-503.

16. Collins WE, Jeffery GM, Roberts JM. A retrospective examination of reinfection of humans with Plasmodium vivax. Am J Trop Med Hyg 2004; 70:642-4.
17. Adak T, Sharma VP, Orlov VS. Studies on the Plasmodium vivax relapse pattern in Delhi, India. Am J Trop Med Hyg 1998; 59:175-9.

18. Haghdoost AA, Mazhari S, Bahaadini K. Estimating the relapse risk of Plasmodium vivax in Iran under national chemotherapy scheme using a novel method. J Vector Borne Dis 2006; 43:168-72.

19. Boulos M, Neto VA, Dutra AP, Disanti SM, Shiroma M. Analysis of the frequency of relapses due to malaria caused by Plasmodium vivax in a nonendemic area (São Paulo, Brazil). Rev Inst Med Trop São Paulo 1991; 33:143-6.

20. Pukrittayakamee S, Chantra A, Simpson JA, Vanijanonta S, Clemens R, Looareesuwan S, et al. Therapeutic responses to different antimalarial drugs in vivax malaria. Antimicrob Agents Chemother 2000; 44:1680-5.

21. Karunajeewa HA, Mueller I, Senn M, Lin E, Law I, Gomorrai PS. A trial of combination antimalarial therapies in children from Papua New Guinea. N Engl J Med 2008; 11:359-83.

22. Tauil PL, Luz FCO, Oliveira APL, Deckers FAL, Santos JB. Malária vivax com tempo de incubação prolongado, detectada no Distrito Federal: relato de três casos. Rev Soc Bras Med Trop 2010; 43:213-4.

23. Hill E, Amatuzio DS. Southwest Pacific vivax malaria: clinical features and observations concerning duration of clinical activity. Am J Trop Med Hyg 1949; 29:203-14.

24. Most $\mathrm{H}$. Clinical trials of anti-malarial drugs. Internal Medicine in World War II 1963; 2:525-98.

25. Gogtay NJ, Desai S, Kadam VS, Kamtekar KD, Dalvi SS, Kshirsagar NA. Relapse pattern of Plasmodium vivax in Mumbai: a study of 283 cases of vivax malaria. J Assoc Physicians India 2000; 48:1085-6.

26. Lysenko AJ, Beljaev AE, Rybalka VM. Population studies of Plasmodium vivax. 1. The theory of polymorphism of sporozoites and epidemiological phenomena of tertian malaria. Bull World Health Organ 1977; 55:541-9.

27. Brasil P, Costa AP, Pedro RS, Bressan CS, Silva S, Tauil PL, et al. Unexpectedly long incubation period of Plasmodium vivax malaria, in the absence of chemoprophylaxis, in patients diagnosed outside the transmission area in Brazil. Malar J 2011; 10:122.

28. Luxemburger C, van Vugt M, Jonathan S, McGready R, Looareesuwan S, White NJ. Treatment of vivax malaria on the western border of Thailand. Trans R Soc Trop Med Hyg 1999; 93:433-8.

29. John GK, Nosten F, Baird K, White NJ, Douglas NM, Seidlein LV, et al. Primaquine radical cure of Plasmodium vivax: a critical review of the literature. Malar J 2012; 11:280.

30. Luxemburger C, Thwai KL, White NJ, Webster HK, Kyle DE, Maelankirri L. The epidemiology of malaria in a Karen population on the western border of Thailand. Trans R Soc Trop Med Hyg 1996; 90:105-11. 
31. Arnott A, Barry AE, Reeder JC. Understanding the population genetics of Plasmodium vivax is essential for malaria control and elimination. Malar J 2012; 10:11.

32. Looareesuwan S, White NJ, Bunnag D, Chittamas S, Harinasuta T. High-rate of Plasmodium vivax relapse following treatment of falciparum malaria in Thailand. Lancet 1987; 2:1052-5.

33. Graves PM, Ngondi JM, Hwang J, Getachew A, Gebre T, Mosher AW. Factors associated with mosquito net use by individuals in households owning nets in Ethiopia. Malar J 2011; 13:10.
34. Doolan DL, Dobano C, Baird JK. Acquired immunity to malaria. Clin Microbiol Rev 2009; 22:13-36.

35. Baird JK. Host age as a determinant of naturally acquired immunity to Plasmodium falciparum. Parasite Immunol 1995; 11:105-11.

Submitted on 06/Dec/2012

Final version resubmitted on 24/Oct/2013

Approved on 26/Nov/2013 